\title{
ATIVIDADES FORA DA NUCLEAÇÃO FAMILIAR: Uma experiência de independência no desempenho das Atividades de Vida Diária vivenciada pela Terceira Idade**
}

\author{
Simone Regina Alves de Freitas BARROS ${ }^{1}$ \\ Pedro Henrique de Barros FALCÃO ${ }^{2}$
}

1. Enfermeira. Secretária Adjunta de Saúde da Secretaria Municipal de Saúde de Buíque Pernambuco; Enfermeira da Estratégia de Saúde da Família do Município de Tupanatinga; Especialista em Saúde Pública e Gestão pela a Universidade Estadual de Pernambuco; Pós-Graduada em Enfermagem Obstétrica pelo Espaço Enfermagem; Mestre em Administração em Organizações e a Saúde do Trabalhador pela Escola Superior Aberta do Brasil e Mestranda em Saúde Pública e Gestão Hospitalar pela Faculdade do Norte do Paraná. Buíque, PE, Brasil.

2. Bacharel em Ciências Biológicas pela Universidade Federal Rural de Pernambuco. Mestre em Ciências em Biologia Celular e Molecular pela Fundação Oswaldo Cruz. Doutorando em Ensino, Filosofia e História das Ciências pela Universidade Federal da Bahia e Universidade Estadual da Paraíba. Professor assistente com dedicação exclusiva da Universidade de Pernambuco. Atualmente acumula as PróReitorias de Administração e de Integração e Fortalecimento da Interiorização da Universidade de Pernambuco.

*UNIVERSIDADE DE PERNAMBUCO - FACULDADE DE CIÊNCIAS, EDUCAÇÃO E TECNOLOGIA DE GARANHUNS - PERNANBUCO.

*FACULDADE DO NORTE DO PARANÁ- INSTITUTO BIOEDUCAÇÃO.

Endereço para correspondência:

Simone Regina Alves de Freitas Barros

Av. Jonas Camelo, 316 - Centro 56520-000 Buíque, PE.

E-mail: simoninhabarros2010@hotmail.com

Pedro Henrique de Barros Falcão

Rua Capitão Pedro Rodrigues, 105 - São José

55294902 - Brasil - Caixa-postal: 15·Garanhuns PE.

E-mail: pedro-falcao@hotmail.com

Recebido em: 28/05/2014 - Aprovado em: 19/09/2014 - Disponibilizado em: 15/12/2014

\section{Resumo}

JUSTIFICATIVA E OBJETIVOS: O Brasil é um país que envelhece a passos largos. Mesmo a velhice sendo um processo universal, apresenta um forte componente de gênero, pois existem mais idosas que idosos. A presente pesquisa teve como objetivo descrever o perfil socioeconômico, demográfico de mulheres idosas (60 anos e mais) inseridas em um programa de terceira idade do SESC Arcoverde estado de Pernambuco e avaliar seu nível de capacidade funcional no desempenho das Atividades de Vida Diária. MÉTODO: Estudo transversal com abordagem descritiva. Realizado com 60 idosas. Ocorreu no período de abril de 2008 a maio de 2012. RESULTADOS: Da população estudada $94 \%$ das idosas são independentes nas Atividades de Vida Diária: banho, vestir-se, ir ao banheiro e alimentar-se. Sendo que um percentual de 7\% relataram acidentes ocasionais de perdas urinárias. CONCLUSÃO: Conclui-se assim, em dizer que as iniciativas privadas como é o caso do SESC e outras entidades que desenvolvem atividades voltadas para a população idosa transformam o envelhecimento em uma experiência mais gratificante e que o trabalho notável dessas entidades é proporcional à precariedade dos mecanismos de saúde pública que dispomos para lidar com os problemas da idade avançada em nosso país.

Descritores: Idosas, AVDs, SESC. 


\title{
ACTIVITIES OUTSIDE THE NUCLEATION FAMILY: an experience of independence in performing ADLs experienced by the Third Age
}

\begin{abstract}
Summary
BACKGROUND AND OBJECTIVES: Brazil is a country that is aging strides . Even old age is a universal process, presents a strong gender component, because there are more elderly than elderly . This study aimed to describe the socioeconomic, demographic profile of older women ( 60 and over ) inserted in a program for the elderly SESC Arcoverde - state of Pernambuco and assess their level of functional capacity in performing activities of daily living. METHODS : Cross-sectional study with descriptive approach . Conducted with 60 elderly . Occurred from April 2008 to May 2012 . RESULTS : In the study population of elderly are $100 \%$ independent in activities of daily living : bathing, dressing , toileting and feeding. As a percentage of $7 \%$ reported occasional urinary leakage accidents . CONCLUSION : We conclude therefore, to say that private initiatives such as the SESC and other entities that develop activities for the elderly transform aging in a more rewarding experience and the outstanding work of these entities is proportional to the precariousness of mechanisms for public health that we have to deal with the problems of old age in our country
\end{abstract}

Keywords: Elderly, ADL, SESC .

\section{INTRODUÇÃO}

Segundo o censo demográfico de 2010, a população brasileira corresponde a 190.755.199 milhões de pessoas, sendo que $51 \%$, o equivalente a 97 milhões, são mulheres e 49\%, o equivalente a 93 milhões, são homens. O contingente de pessoas idosas, que, segundo a Política Nacional do Idoso e o Estatuto do Idoso, tem 60 anos a mais, é de 20.590.599 milhões, ou seja, aproximadamente $10,8 \%$ da população total. Desses, 55,5 \% (11.434.487) são mulheres e 44,5\% (9.156.112) são homens ${ }^{1}$. Uma análise das condições de vida da população brasileira em 2012 mostrou que o ritmo de envelhecimento da população brasileira está acima da média mundial. A conclusão tem como base o índice de envelhecimento do país, calculado por meio da razão entre o número de pessoas de 60 anos ou mais de idade para cada 100 pessoas de menos de 15 anos de idade ${ }^{2}$.

A Organização das Nações Unidas (ONU) considera o período de 1975 a 2025 como a Era do Envelhecimento. Enquanto nas nações desenvolvidas, no período de 1970 a 2000, o envelhecimento populacional observado foi de 54\%, nos países em desenvolvimento atingiu $123 \%{ }^{3}$.

O Brasil é um país que envelhece a passos largos. As alterações na dinâmica populacional são claras, 
inequívocas e irreversíveis. Desde os anos 1940, é entre a população idosa que temos observado as taxas mais altas de crescimento populacional. Na década de 50, a taxa de crescimento da população idosa atingiu valores superiores a $3 \%$ ao ano, chegando a 3,4\%, entre 1991 e 2000. Ao compararmos, num intervalo de 25 anos (1980 a 2005), o crescimento da população idosa com o crescimento da população total, observamos que o crescimento da população idosa foi de $126,3 \%$, ao passo que o crescimento da população total foi de apenas 55,3\%. Nesse mesmo intervalo, o segmento de 80 anos a mais cresceu a um ritmo relativamente maior do que a população idosa total, apresentando um crescimento de $246,0 \%$. Hoje, a faixa etária de 80 anos a mais é composta por 2.935.585 pessoas (IBGE, 2011), representando $14 \%$ da população idosa brasileira ${ }^{1}$. Observando os dados da atual transição demográfica brasileira sob a ótica de gênero, constatamos um processo de feminização da velhice, ou seja, quanto mais a população envelhece, mais feminina ela se torna. Hoje, as mulheres representam 55,5\% da população idosa brasileira e $61 \%$ do contingente de idosos acima de 80 anos $\left(\right.$ IBGE, 2011) ${ }^{1}$. Haverá assim, em meados do século, quase duas mulheres para cada homem entre os mais idosos ${ }^{4}$. Essa sobre representação feminina resulta da maior expectativa de vida das mulheres que, em média, vivem 8 anos a mais que os homens ${ }^{1}$ Com o envelhecimento, as mulheres são afetadas diferentemente de homens, tornando-as mais vulneráveis não apenas aos problemas de saúde, mas ao isolamento social e a transtornos emocionais devido à aposentadoria, à viuvez, às alterações fisiológicas, dentre outros problemas $^{1}$. No Brasil, estima-se que $10 \%$ das pessoas acima de 65 anos precisam de ajuda em atividades básicas de autocuidado e cerca de $40 \%$ necessitam de auxílio nas atividades instrumentais de vida diária, o que significa mais de 6 milhões de pessoas precisando de suporte ${ }^{1}$.

Nesse sentido, em julho de 1995, o Programa de Atenção Integral à Saúde do Idoso que foi elaborado em consonância à Lei 8.842/94, regulamentada em julho de 1996, essa dispõe sobre a Política Nacional do Idoso. Tal legislação tem como propósito basilar a promoção do envelhecimento saudável, a manutenção e a melhoria, ao máximo, da capacidade funcional dos idosos, a prevenção de doenças, a recuperação da saúde dos que adoecem e a reabilitação daqueles que venham a ter a sua capacidade 
funcional restringida, de modo a garantir-lhes permanência no meio em que vivem exercendo de forma independente suas funções na sociedade ${ }^{5}$.

A capacidade funcional surge como um novo paradigma de saúde, particularmente um valor ideal para que o idoso possa viver independente, sendo esta a capacidade do indivíduo realizar suas atividades físicas e mentais necessárias para manutenção de suas atividades básicas, ou seja, tomar banho, vestir-se, realizar higiene pessoal, transferir-se, alimentar-se, manter a continência. A manutenção e a preservação da capacidade para desempenhar as atividades básicas de vida diária são pontos básicos para prolongar o maior tempo possível à independência ${ }^{6}$.

Os idosos, principalmente as mulheres, apresentam altas taxas de dependência e declínio da capacidade funcional, o que as leva a maior fragilidade, perda da autonomia e acaba impedindo-as de realizarem suas atividades cotidianas ${ }^{7}$.

No entanto, é dentro desse contexto que a denominada "avaliação funcional" torna-se essencial para o estabelecimento de um diagnóstico, um prognóstico e um julgamento clínico adequados, que subsidiarão as decisões sobre os tratamentos e cuidados necessários a pessoa idosa, seja no ambiente clínico, grupos sociais ou centro de convivência. É um parâmetro que, associado a outros indicadores de saúde, pode ser utilizado para determinar a eficácia e a eficiência das intervenções propostas ${ }^{8}$. Cabe ressaltar que certas alterações decorrentes do processo de envelhecimento podem ter seus efeitos minimizados pela assimilação de um estilo de vida mais ativo ${ }^{9}$.

Parece-nos, portanto, bastante relevante conhecer o perfil dessas idosas. Uma vez que empresa (SESC) desenvolve ações voltadas para a valorização do cidadão idoso, visando sempre a promoção para o envelhecimento saudável. Como também, discutir-se e divulgar para a sociedade o impacto das atividades sociais fora da nucleação familiar como fator preditivo de envelhecimento saudável, mediante a assimilação de um estilo de vida mais ativo. Sendo assim, o objetivo desse estudo foi descrever o perfil socioeconômico, demográfico de mulheres idosas inseridas no programa de terceira idade do Serviço Social do Comércio (SESC) de Arcoverde Pernambuco (PE) e avaliar seu nível de capacidade funcional 
desempenho das Atividades Básicas de

\section{MÉTODO}

Estudo transversal de natureza (1963), contendo dezoito questões quantitativa e com abordagem descritiva. No qual foram avaliadas idosas pertencentes a um grupo de Terceira Idade de uma empresa privada. O estudo aconteceu na Unidade do SESC de Arcoverde-PE, empresa mantida pelos empresários do comércio de bens e serviços, essa é uma entidade voltada para o bem-estar social de sua clientela. $\mathrm{O}$ estudo decorreu no período de abril de 2008 a maio de 2012.

A população da amostra foram 60 mulheres idosas, com 60 anos e mais, cadastradas em um programa para Terceira Idade do SESC - Arcoverde PE. Perfazendo um total de $100 \%$ das idosas cadastradas. Essas assinaram o Termo de Consentimento Livre e Esclarecido após esclarecimentos dos pesquisadores sobre os objetivos da pesquisa.

instrumento de coleta deu-se através de uma entrevista estruturada, a partir de um roteiro adaptado do Formulário de Avaliação das Atividades Básicas de Vida Diária (AVDs) de Sidney Katz dissertativas. Portanto, a pontuação de dependência e de independência foi obtida mediante o Index de AVDs de Katz (1976) que é a escala utilizada para padronização desse tipo de estudo.

Os dados foram digitados em planilhas do Excel - Windows XP que possibilitou a organização dos dados em tabelas para melhor interpretação dos resultados. A análise estatística deu-se mediante a importação dos dados da versão Excel para o programa SPSS (Statistiscal Package for the Social Sciences) na versão 13. Os dados obtidos refletem um grau de confiabilidade estatística de 95\%. A pesquisa foi desenvolvida, conforme as normas da resolução 196 de outubro de 1996 do Conselho Nacional de Saúde. O projeto foi cadastrado no Sistema Nacional de Informação sobre Ética em Pesquisa (SISNEP) e encaminhado ao Comitê de Ética da Universidade Estadual de Pernambuco. Tendo obtido sua aprovação através do protocolo CAAE - 0036.0.097.000-09. 


\section{RESULTADOS}

Levando-se em consideração o perfil socioeconômico e demográfico das idosas e o grau de capacidade funcional dessas, as tabelas a seguir versam sobre as assertivas de maior relevância para a compreensão dos questionamentos que direcionaram os objetivos desta pesquisa.

A tabela 1 foi distribuída mediante categorias cronológicas. Visto que $67 \%$ das idosas estavam na faixa etária de 60 a 69 anos, $6 \%$ compreendiam a faixa etária de 70 a 79 anos e por fim, $27 \%$ estavam representando a categoria dos 80 e mais.

Tabela 1 - Distribuição das idosas do Programa de Terceira Idade do SESC Arcoverde-PE segundo a variável idade (2012)

\begin{tabular}{c|c}
\hline Idade & \% \\
\hline $60-69$ & $67 \%$ \\
$70-79$ & $6 \%$ \\
$80+$ & $27 \%$ \\
\hline Total & $\mathbf{1 0 0 \%}$ \\
\hline
\end{tabular}

Quanto à escolaridade, conforme podemos visualizar na tabela 2 verificou-se que $33 \%$ das idosas concluíram o ensino superior. Em contrapartida $33 \%$ não chegaram a concluir o ensino fundamental, apenas cursaram o antigo primário.
Tabela 2 - Distribuição da variável escolaridade das Idosas do Programa de Terceira Idade do SESC-Arcoverde-PE/2012

\begin{tabular}{l|c}
\hline \multicolumn{1}{c|}{ Escolaridade } & \% \\
\hline Antigo Primário & $33 \%$ \\
Fundamental & $20 \%$ \\
Médio & $14 \%$ \\
Superior & $33 \%$ \\
Não Alfabetizada & $0 \%$ \\
\hline Total & $\mathbf{1 0 0 \%}$ \\
\hline
\end{tabular}

Conforme versa a tabela 3 observou-se que $80 \%$ das idosas são aposentadas.

Tabela 3 - Distribuição das idosas cadastradas no PTI do SESC mediante Situação Previdenciária em 2012

\begin{tabular}{|c|c|}
\hline Situação Previdenciária & $\%$ \\
\hline Aposentada & $80 \%$ \\
\hline Pensionista & $20 \%$ \\
\hline Total & $100 \%$ \\
\hline
\end{tabular}

Com relação à estrutura familiar do grupo de idosas aqui pesquisadas, conforme pode ser visualizado na tabela 4 observou-se que $73 \%$ das idosas moram com filhos e $20 \%$ com filhos e esposo. Sendo que um percentual pequeno $7 \%$ moram sozinhas. 
Tabela 4 - Distribuição das idosas cadastradas no Programa de Terceira Idade do SESCArcoverde segundo a Estrutura Familiar em 2012

\begin{tabular}{c|c}
\hline Composição Familiar & $\mathbf{\%}$ \\
\hline Filhos & $73 \%$ \\
Esposo \& Filhos & $20 \%$ \\
Esposo & $0 \%$ \\
Netos & $0 \%$ \\
Outros & $0 \%$ \\
Sozinha & $\mathbf{7 \%}$ \\
\hline Total & $\mathbf{1 0 0 \%}$ \\
\hline
\end{tabular}

Levando-se em consideração os resultados apresentados na tabela 5, constatou-se que $40 \%$ das idosas dispõem de uma renda mensal de até um salário mínimo. As demais possuem uma renda mensal que varia entre 2 e 4 salários mínimos.

Tabela 5 - Distribuição de Renda Mensal das Idosas cadastradas no Programa de Terceira Idade do SESC Arcoverde-PE em 2012

\begin{tabular}{c|c}
\hline Renda Mensal & \% \\
\hline Até 1 salário mínimo & $40 \%$ \\
2 salários mínimos & $20 \%$ \\
3 salários mínimos & $7 \%$ \\
4 salários mínimos & $20 \%$ \\
5 salários mínimos & $7 \%$ \\
+ de 5 salários mínimos & $\mathbf{1 0 0 \%}$ \\
\hline \multicolumn{2}{c}{ Total } \\
\hline A tabela 6 traz uma síntese sobre o hábito \\
frequente de praticar atividade física, uso de \\
tabaco, consumo de álcool e convênio de
\end{tabular}

saúde. Conforme visualizados os dados a seguir as idosas que praticam atividades físicas regularmente decorrem em um percentual de 73\%. Enquanto 93\% não têm o hábito de fumar. Já $87 \%$ afirmam não fazer com frequência o uso de álcool. Entretanto $60 \%$ das idosas não têm plano privado de saúde.

Tabela 6 - Distribuição de idosas cadastradas no Programa de Terceira Idade do SESC Arcoverde-PE em 2012 segundo as variáveis atividade física, hábito de fumar e uso de álcool

\begin{tabular}{l|c|c|c|c}
\cline { 2 - 5 } & $\begin{array}{c}\text { Atividade } \\
\text { Física } \\
\text { Regular }\end{array}$ & $\begin{array}{c}\text { Hábito } \\
\text { fe } \\
\text { Fumar }\end{array}$ & $\begin{array}{c}\text { Uso de } \\
\text { álcool }\end{array}$ & $\begin{array}{c}\text { Convênio } \\
\text { de Saúde } \\
\text { (Privado) }\end{array}$ \\
\hline Sim & $73 \%$ & $7 \%$ & $13 \%$ & $\mathbf{4 0 \%}$ \\
\hline Não & $27 \%$ & $93 \%$ & $87 \%$ & $\mathbf{6 0 \%}$ \\
\hline Total & $\mathbf{1 0 0 \%}$ & $\mathbf{1 0 0 \%}$ & $\mathbf{1 0 0 \%}$ & $\mathbf{1 0 0 \%}$ \\
\hline
\end{tabular}

A Tabela 7 versa sobre auto avaliação das idosas acerca do nível de independência ou não perante a realização das Atividades Básicas de Vida Diária. Os resultados demonstram um alto nível de independência na somatória de todas as atividades (94\%). Sendo que apenas as atividades transferência e continência obtiveram respectivamente menores percentuais de independência (93\% e 73\%). As demais AVDs apresentaram uma independência de 100\% (banho, vestir, ir ao banheiro e alimentação). 
Tabela 7 - Distribuição das idosas cadastradas no PTI do SESC-Arcoverde segundo a realização das Atividades Básicas de Vida Diária-AVDs em 2012

\begin{tabular}{|c|c|c|c|}
\hline \multicolumn{2}{|c|}{ AVDs } & \multicolumn{2}{|c|}{$\%$} \\
\hline \multicolumn{2}{|l|}{ Banho } & \multicolumn{2}{|c|}{$100 \%$} \\
\hline \multicolumn{2}{|l|}{ Vestir } & \multicolumn{2}{|c|}{$100 \%$} \\
\hline \multicolumn{2}{|l|}{ Ir ao Banheiro } & \multicolumn{2}{|c|}{$100 \%$} \\
\hline \multicolumn{2}{|l|}{ Transferência } & \multicolumn{2}{|c|}{$93 \%$} \\
\hline \multicolumn{2}{|l|}{ Continência } & \multicolumn{2}{|c|}{$73 \%$} \\
\hline \multicolumn{2}{|l|}{ Alimentação } & \multicolumn{2}{|c|}{$100 \%$} \\
\hline \multirow[t]{2}{*}{ AVDs } & \multicolumn{3}{|c|}{ FAIXAS ETÁRIAS } \\
\hline & $60-69$ & $70-79$ & $80+$ \\
\hline Banho & $100 \%$ & $100 \%$ & $100 \%$ \\
\hline Vestir & $100 \%$ & $100 \%$ & $100 \%$ \\
\hline Ir ao banheiro & $100 \%$ & $100 \%$ & $100 \%$ \\
\hline Transferência & $100 \%$ & $100 \%$ & $7 \%$ \\
\hline Continência & $7 \%$ & $7 \%$ & $7 \%$ \\
\hline Alimentação & $100 \%$ & $100 \%$ & $100 \%$ \\
\hline & $\mathrm{n}=60$ & $\mathrm{n}=60$ & $\mathrm{n}=60$ \\
\hline
\end{tabular}

A tabela 8 enumera mediante o Index de AVDs de Kartz de 0 a 6 o grau de independência de um indivíduo na realização de tais atividades e, indiretamente, o estado de saúde e a necessidade de auxílio. $\mathrm{O}$ index 0 apresentou um percentual de $67 \%$, esse percentual de independência está distribuído para todas as AVDs mediante essa classificação de Kartz. Ainda pode ser observado que $33 \%$ da população de idosas demonstram uma classificação 1 no Index de AVDs.

\section{DISCUSSÃO}

De acordo com a tabela 1 observou-se que há uma predominância de idosas na faixa etária de 60 a 69 anos de idade
Tabela 8 - Distribuição das idosas cadastradas no Programa de Terceira Idade do SESCArcoverde-PE segundo o Index de AVDs $(\text { Kartz) })^{8}$ em 2012

\begin{tabular}{|c|c|c|}
\hline Tipo de classificação & $\begin{array}{l}\text { Index de } \\
\text { AVDs } \\
\text { (Katz) }\end{array}$ & $\%$ \\
\hline $\begin{array}{l}\text { Independente nas seis } \\
\text { funções (banhar-se, } \\
\text { vestir-se, } \\
\text { alimentação, ir ao } \\
\text { banheiro, } \\
\text { transferência } \\
\text { continência) }\end{array}$ & 0 & $67 \%$ \\
\hline $\begin{array}{l}\text { Independente } \text { em } \\
\text { cinco funções e } \\
\text { dependente em uma } \\
\text { função }\end{array}$ & 1 & $33 \%$ \\
\hline $\begin{array}{l}\text { Independente } \text { em } \\
\text { quatro funções e } \\
\text { dependente em duas } \\
\text { funções }\end{array}$ & 2 & 0\% \\
\hline $\begin{array}{l}\text { Independente em três } \\
\text { funções e dependente } \\
\text { em três funções }\end{array}$ & 3 & $0 \%$ \\
\hline $\begin{array}{lr}\text { Independente } & \text { em } \\
\text { duas funções } & \mathrm{e} \\
\text { dependente } & \mathrm{em} \\
\text { quatro funções } & \end{array}$ & 4 & 0\% \\
\hline $\begin{array}{l}\text { Independente } \\
\text { uma função em } \\
\text { dependente em cinco } \\
\text { funções }\end{array}$ & 5 & $0 \%$ \\
\hline $\begin{array}{l}\text { Dependente para } \\
\text { todas as funções }\end{array}$ & 6 & $0 \%$ \\
\hline
\end{tabular}

cadastradas no PTI. Outro estudo interdisciplinar com 40 idosos socialmente ativos (frequentadores de atividades fora do núcleo familiar) mostrou que pouco mais da metade da 
amostra também feminina possuíam entre 60 e $69 \operatorname{anos}^{10}$.

As idosas pesquisadas têm em média cinco a dez anos de participação nas atividades sociais oferecidas pela empresa SESC. O grau de escolaridade das idosas reflete em um nível bom para realidade brasileira, pois $33 \%$ dessas chegaram a concluir o ensino superior, todas as demais obtiveram um nível de educação formal. Uma pesquisa sobre o perfil socioeconômico e demográfico dos idosos brasileiros declarou que $89 \%$ não passaram da $8^{\mathrm{a}}$ série do ensino fundamental $18 \%$ não tiveram nenhuma educação formal e apenas $4 \%$ chegaram ao $3^{\circ}$ grau (ensino superior) de escolaridade (completo ou incompleto) $^{11}$.

Conforme podemos visualizar na tabela 4 em termos de estrutura familiar observou-se que um grande percentual de idosas moram com filhos $(73 \%)$ e apenas $20 \%$ com filhos e esposo. De acordo com um estudo sobre a estrutura familiar brasileira mostrou que está tendo uma redução do número de membros da família. Esse fenômeno ocorre paralelamente ao aumento da participação dos idosos na população geral $^{12}$.

A renda mensal das idosas conforme pode ser visualizada na tabela 5 está distribuída entre 1 a 5 ou mais salários mínimos. Sendo que $40 \%$ recebem mensalmente uma renda de até um salário mínimo. As demais idosas dispõem de uma renda que varia entre 2 e 4 salários mínimos. Estudo relata que economicamente, a imensa maioria dos idosos $(82,9 \%)$ recebe no máximo até 2 salários mínimos ${ }^{13}$.

Observou-se ainda neste estudo (tabela 6) que $73 \%$ das idosas praticam atividades físicas regularmente com uma frequência de 3 a 4 vezes por semana. A atividade física de maior prevalência foi à hidroginástica com um percentual de 53\% seguido da caminhada com 20\%. Em um estudo com a terceira idade, constatou-se que $56 \%$ dos idosos referiram praticar atividades físicas regularmente ${ }^{14}$. Ainda de acordo com a tabela 6 observou-se que um elevado percentual de idosas (93\%) não tem o hábito de fumar. Pesquisas mostram que o hábito de fumar apresenta um importante papel no desenvolvimento de outras doenças que são frequentes na senilidade como demência, osteoporose, diabetes, úlcera péptica, refluxo gastresofágico, disfunção erétil, degeneração macular relacionada à idade, catarata nuclear, alterações de audição, além de lesões cutâneas ${ }^{15}$. 
Na tabela 7 é possível observar que na auto avaliação das idosas com base na capacidade de execução das AVDs, que por sua vez se dividem em: atividades básicas da vida diária - tarefas próprias do autocuidado para banho, vestir-se, ir ao banheiro e alimentar-se. Os percentuais apresentados não corroboram com outros estudos nesta linha de pesquisa, em que o nível de independência para realização das AVDs foi de apenas $41,8 \%{ }^{13}$. Em outra pesquisa nacional realizada no município de São Paulo evidenciou-se que mais da metade da população estudada $(53 \%)$ referiam necessidades de ajuda parcial ou total para realizar pelo menos uma das atividades da vida diária. Foi detectado também, em outro estudo que $29 \%$ dos idosos necessitavam de ajuda parcial ou total para realizar até três dessas atividades, e $17 \%$ necessitavam de ajuda para realizar quatro ou mais atividades da vida diária $^{16}$. Segundo a classificação do index 1 a tabela 8 mostra o percentual de dependência por faixa etária, nessa observou-se que o index de dependência de uma atividade não está relacionado com a faixa etária das idosas e sim com prováveis doenças de base ou manifestações comuns ao processo de envelhecimento. Conforme decorrem os números percentuais todas as faixas etárias relataram acidentes ocasionais de perdas urinárias (7\%). Mediante um percentual pouco expressivo segundo a divisão cronológica apresentada no estudo. Em outro estudo, 37,6\% dos idosos referiram já ter perdido urina involuntariamente, sendo $27 \%$ de forma eventual e 7,5\% de forma frequente. Dados esses que não corroboram com essa pesquisa. Pois segundo a auto avaliação das idosas há perdas eventuais de urina e não de forma frequente ${ }^{14}$.

A prevalência de Incontinência Urinária (IU) é maior em mulheres que nos homens entre 50 e 75 anos, não apresentando variações por sexo na idade mais avançada. Estima-se que entre a população idosa a prevalência de IU é de aproximadamente 10 a $15 \%$ entre os homens e 20 a $35 \%$ entre as mulheres. Nas mulheres, a principal alteração é a redução da pressão máxima de fechamento uretral, consequência de danos secundários a partos, cirurgias, radiação, tabagismo, obesidade, distúrbios neurológicos, da redução da vascularização e hipotrofia dos tecidos que revestem e envolvem a uretra, a bexiga e a vagina e outros ${ }^{17}$.

Estudos mostram que muitas mulheres passam a conviver com perdas mínimas de urina durante muito tempo sem se queixarem entendendo que isso é 
"normal". A intensidade varia desde pequenas perdas, durante esforços súbitos que aumentem a pressão intraabdominal como tossir ou espirrar até a incontinência completa com esvaziamento total do conteúdo vesical e/ou fecal. Calcula-se que pelo ou menos $1 / 3$ da população idosa americana tenha algum grau de dificuldade em controlar a urina ${ }^{18}$.

As idosas de 80 anos e mais apresentaram um pequeno percentual

\section{CONSIDERAÇÕES FINAIS}

A Terceira Idade é uma das mais importantes etapas da vida, talvez a mais difícil, pois com ela vêm as mudanças fisiológicas, biológicas e principalmente a psicológica. Para cada pessoa, o sentimento do envelhecimento é muito diferente.

Contudo,

a imagem do envelhecimento na atualidade quando associado às políticas atuais de saúde do idoso, não oferece instrumentos capazes de enfrentar os problemas envolvidos na perda de habilidades cognitivas e de controles físicos e emocionais que estigmatizam o velho e que são fundamentais, na sociedade, para que um indivíduo seja reconhecido como um ser autônomo,
7\% de dependência com relação à transferência, ou seja, essas idosas relataram que ao deitar e levantar-se da cama ou cadeira necessitam de um objeto de apoio. Dados de uma pesquisa transversal indicam que há uma perda aproximada de $20 \%$ na força por volta dos 60 anos de idade e uma perda de força mais drástica de $40 \%$ entre 70 e 80 anos de idade ${ }^{19}$.

capaz de um exercício pleno dos direitos de cidadania.

Para alguns estudiosos o envelhecimento populacional é um dos maiores desafios da saúde pública contemporânea, atrelada a essa realidade é de suma importância mostrar aos formuladores e gestores das políticas sociais, o cenário de indicadores de saúde dessa população, como também, as iniciativas de sucesso para o envelhecimento saudável. Há, portanto um imperioso discurso acerca de soluções que até o momento não evoluíram ao ritmo da velocidade do crescimento desta população. Sendo necessário, à implementação de soluções atingíveis para as diversas questões concernentes ao processo de 
envelhecimento com efeitos minimizados pela assimilação de um estilo de vida diferenciado.

É obvio que a sistematização do cuidado preconizada na Política de Saúde do Idoso privilegia a promoção da saúde, à prevenção de incapacidades e à manutenção da capacidade funcional como sendo de fundamental importância e insubstituíveis. Mas que atrelado a essa política, a dependência precisa ser reconhecida como uma importante questão de saúde pública.

A auto avaliação das idosas com base na capacidade de execução das AVDs apresentou uma fotografia diferenciada de estudos nacionais e internacionais. Uma vez que essas obtiveram um elevado grau de independência mediante o desempenho de suas atividades de vida cotidiana. Dentro desse contexto, as iniciativas privadas como é o caso do SESC e outras entidades que desenvolvem atividades voltadas para a população idosa transformam o envelhecimento em uma experiência mais gratificante, contudo, esse trabalho notável dessas entidades é proporcional à precariedade dos mecanismos de saúde pública que dispomos para lidar com os problemas da velhice avançada.

Em resposta à necessidade de transformação na gestão de saúde da população idosa nos diversos seguimentos sociais, a pesquisa aqui apresentada descreve a experiência de um grupo de idosas diferenciado das literaturas correlatas. Essas idosas têm uma vida socialmente ativa e participam de atividades fora da nucleação familiar há mais de 10 anos no SESC. Observou-se ainda, que as atividades vivenciadas pelo grupo vêm contribuindo para um elevado nível de independência. Mesmo a amostra tratando de uma população do sexo feminino, que segundo as evidências científicas têm uma maior probabilidade de desenvolver dependência funcional, foi possível então, mostrar um novo perfil dessa população.

Por fim, esta pesquisa busca novas linhas de fuga para pensarmos na saúde do idoso a partir de práticas sociais que valorize $\mathrm{o}$ idoso nos seus mais diferentes aspectos socioeconômicos e culturais. 


\section{REFERÊNCIAS}

1- Kuchemann, BA. Envelhecimento populacional, cuidado e cidadania: velhos dilemas e novos desafios. Soc. estado. 2012, 1(27): 165-180.

3- Melo, M CS et al., A educação em saúde como agente promotor de qualidade de vida para o idoso. Ciênc. saúde coletiva.2009; 1(14): 15791586.

2- Saraiva A; Martins D. População do país envelhece em ritmo acima da média mundial, diz IBGE. Valor Econômico. Disponível em: <http://www.valor.com.br/brasil/29198 94/populacao-do-pais-envelhece-emritmo-acima-da-media-mundial-dizibge> . Acesso em: 06 de julho de 2013.

4- Carvalho, JAM; Wong, LR. A transição da estrutura etária da população brasileira na primeira metade do século XXI. Cad. Saúde Pública, Rio de Janeiro,2008; 3(24):597-605.

5-Politíca Nacional do Idoso. Disponível em:< www.jusbrasil.com.br/topicos/.../lei-n8842-de-04-de-janeiro-de-1994>. Acesso em: 02 de outubro de 2013.

6- Guimarães, LHCT, et al. Avaliação da capacidade funcional de idosos em tratamento fisioterapêutico. Revista Neurociências, São Paulo. 2004; (3) 12:130-133.

7- Paz, AA; Santos, BR L; Eidt, OR. Vulnerabilidade e envelhecimento no contexto da saúde. Acta Paul Enferm. 2006; 3(19): 338-342.
8- Duarte, YAO; Andrade, CL; Lebrão ML. O Index de Katz na avaliação da funcionalidade dos idosos. Revista Escola de Enfermagem USP. 2007; 2( 41): 317-325.

9 - Brasil. Ministério da Saúde. Caderno de Atenção Básica. Envelhecimento e Saúde da Pessoa Idosa. Brasília: Ministério da Saúde, 2006.

10- Wathier, JL et al., Eventos de vida e estratégias de coping de idosos socialmente ativos. Estudo interdisciplinar sobre envelhecimento. Porto Alegre. 2007; 1(12): 35-52.

11- Perfil Sociodemográfico dos Idosos Brasileiros em 2007. Disponível em: <http://www2.fpa.org.br/portal/modules /news/article.php?storyid=3516>.Acess o em: 25 de jan. de 2009.

12- Figueiredo, N; Tonini, T, 2009. SUS e PSF para enfermagem - práticas para o cuidado em saúde coletiva. São Caetano do Sul, SP: Yendis Editora, 2007.

13- Alves et al., Perfil da Capacidade Funcional do Idoso. Trabalho apresentado no XVI Encontro Nacional de Estudos Populacionais, realizado em Caxambu-MG - Brasil, de 29 de setembro a 03 de outubro de 2008. Disponível em:<http://www.abep.nepo.unicamp.br/ encontro2008/docspdf/ABEP2008_153 4.pdf>Acesso em : 08 de jan. de 2009.

14 - Anderson, MIP et al., Saúde e Qualidade de Vida na Terceira Idade. Revista Textos Envelhecimento. Rio de Janeiro. 1998; (1) 1: 23-43. 
15- Lima, R. Fumo, saúde e envelhecimento. Disponível em: <http://www.medcentercom/Medscape/ content.aspx?LangType $=1046 \&$ menu_i $\mathrm{d}=49 \& \mathrm{id}=13014>$. Acesso em: $07 \mathrm{de}$ jan. de 2009.

16 - Rosa, TE; Benício, MH; Latorre, MR. Determinantes factors of funcional status among the elderly. Revista de Saúde Pública. Goiás; 1(37): 9-

17- Brasil. Ministério da Saúde. Caderno de Atenção Básica. Envelhecimento e Saúde da Pessoa Idosa. Brasília: Ministério da Saúde, 2006.
18- Incontinência. Disponível em: <http://www.envelhecer comsaude.com.br/index.php?optio $\mathrm{n}=\mathrm{com} \_$content $\&$ task $=$ view $\& \mathrm{id}=85$ $\&$ Itemid=1>. Acesso em: 28 de fev. de 2009.

19 - Assis, EL; Rabelo, HT. Percepção da capacidade funcional de mulheres idosas praticantes de hidroginástica. MOVIMENTUM - Revista Digital de Educação Física. Ipatinga, .2006;1. Disponível em: http://www.unilestemg.br/movimentum/ Artigos_V1N1_em_pdf/movimentum_a ssis_elizetelopes.pdf. Acesso em: 20 de fev de 2012. 